\title{
Energy Deposition During Electron-Induced Dissociation
}

\author{
James R. Gord*, Stevan R. Horning ${ }^{\dagger}$ Joseph M. Wood, R. Graham Cooks, \\ and Ben S. Freiser \\ Department of Chemistry, Purdue University, West Lafayette, Indiana, USA
}

\begin{abstract}
We report studies of the internal energy deposited during activation of mass-selected ions through electron-ion collisions. Characteristic fragmentations of the molecular ion of limonene and $W(\mathrm{CO})_{n}^{+}(n=1-6)$ indicate that electron-induced dissociation in a Fourier transform ion cyclotron resonance mass spectrometer proceeds via multiple collisions and that the average internal energy deposited during the activation process can be selected to be similar to that associated with electron-impact ionization. Control of the degree of ion excitation through selection of the electron energy, flux, and interaction time with the ions of interest is demonstrated, and advantages of this promising activation technique are discussed. () Am Soc Mass Spectrom 1993, 4, 145-151)
\end{abstract}

$\mathrm{T}$ The information contained in a mass spectrum represents a convolution of energy-dependent rate constants for the various unimolecular reactions available to the ions under study over the time scale of the experiment and over the internal energy distributions of the ions [1,2]. Some of the most powerful tools available to the experimentalist using mass spectrometry to investigate the structure of ions and the mechanistic and dynamic details of their chemistry, involve controlled activation of preselected ions in which the internal energy deposited in the ions, and often even the time scale of the experiment, can be manipulated. With the advent of techniques for generating ions of large biomolecules and polymers [3-6], which are often difficult to activate to achieve subsequent dissociation $[7,8]$, a diverse group of methods for producing diagnostic fragmentation is particularly essential.

In working toward this goal, a number of techniques have been developed for activating gaseous ions in tandem mass spectrometry (MS/MS) experiments. These include collision-induced dissociation (CID) [9], surface-induced dissociation (SID) [10], and photodissociation [11]. Each of these methods has been demonstrated in conjunction with Fourier transform ion cyclotron resonance mass spectrometry (FTCRMS) [12-14]. An additional technique that will see application in FTICRMS is based on the characteristic fragmentation produced via endothermic ion-molecule

\footnotetext{
"Present address: Systems Research Lab., 2800 Indian Ripple Road, Dayton, OH 45440-3696.

Present address: Fakultat fur Physik, Universitat-Freiburg, Hermann-Herder-Strasse 3, 7800 Freiburg, Germany.

Address reprint requests to Ben C. Freiser, Department of Chemistry, Purdue University, West Lafayette, IN 47907.
}

reactions [15]. Collision-induced dissociation is the most frequently used technique in FTICR MS/MS experiments; however, this powerful activation tool, as applied in FTICRMS, has a number of significant limitations. The increased pressure associated with the target gas can significantly restrict the mass resolution of the FTICRMS. Dual-cell FTICRMS instruments have been developed to spatially separate high-resolution mass analysis from relatively high-pressure ionization source or ion activation conditions [16, 17]; however, the large radii associated with parent ions excited for CID and the product ions generated as a result of the collision process complicate efficient transfer of these ions between the cells. Despite these difficulties, highresolution CID spectra have been obtained by using the dual-cell configuration [18]. In addition, pulsedvalve introduction of target gases has been used to temporally separate the relatively high- and lowpressure conditions required for efficient CID and high-resolution detection, respectively [19]. A less tractable difficulty is that CID of larger ions ( $>2500$ $\mathrm{Da}$ ) becomes increasingly difficult as the center-of-mass collision energy available for dissociation diminishes $[7,8,20]$.

An alternative technique for ion activation that holds promise for addressing many of these concerns is based on the interaction of energetic electrons with the selected ions. A number of early studies demonstrated the potential of electron-ion interactions for studying energy-resolved second ionization cross sections of atomic ions [21-23] and dissociation cross sections of diatomic ions [24-26]. These principles have been further applied to induce dissociation of polyatomic organic ions [27-33] and $\mathrm{Fe}(\mathrm{CO})_{5}^{+}$[34] by using a technique referred to as electron-impact excitation of 
ions from organics (EIEIO) or electron-induced dissociation (EID). In a recent application of this technique, Wang and McLafferty [20] have demonstrated that EIEIO can produce diagnostic fragmentation of large biomolecular ions such as gramicidin S.

One of the most powerful aspects of any activation technique is the experimentalist's ability to control the amount of energy deposited in the ion of interest during excitation. lon kinetic energy, scattering angle, collision conditions (single or multiple), and target gas selection afford this control in CID. The energy deposited during SID is dependent on the ion kinetic energy and the effective mass of the surface. Control of energy deposition in photodissociation is achieved in a straightforward fashion through selection of the wavelength and the fluence of the excitation source. Similarly, activation is expected to be controlled in EID through manipulation of the electron energy and flux and the interaction time with the ions of interest.

Effective experimental control of the degree of excitation achieved during ion activation requires a thorough understanding of the effects of these parameters on the distribution $P(\epsilon)$ of internal energies $\epsilon$ deposited in the ions. Much of our detailed information concerning the internal energy distributions associated with activated polyatomic ions is based on the work performed with "thermometer molecules," such as various metal carbonyls, tetraethylsilane, and $n$ butylbenzene, which exhibit well-defined fragmentation patterns for which excellent energy-resolved breakdown data have been accumulated. In particular, the fragmentation of several metal carbonyl ions has been used to characterize the deposition of internal energy during electron-impact ionization [35], chargeexchange ionization [35], high- and low-energy CID $[35,36]$, angle-resolved mass spectrometry (ARMS) $[37,38]$, SID [39], and charge stripping [40].

In this study we report the internal energy distribution associated with ions activated during EID on the basis of an analysis of the fragments observed from the limonene molecular ion (Structure 1) and the ions $\mathrm{W}(\mathrm{CO})_{n}^{+}(n=1-6)$. These experiments indicate that there exist similarities between the energy deposited during electron-impact ionization of neutral molecules and EID of preselected ions, suggesting that EID may be a powerful tool for inducing electron-impact-like fragmentation in large molecular ions.

\section{Experimental}

The details of FTICRMS operation have been described previously [41]. All experiments were performed by using a Nicolet FTMS-2000 FTICR mass spectrometer

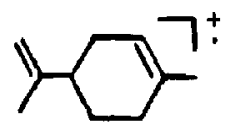

configured with a single rectangular trapping cell $(4.76 \mathrm{~cm} \times 4.76 \mathrm{~cm} \times 9.52 \mathrm{~cm})$ situated in the bore of a 3-T superconducting magnet. The trapping voltage was maintained at $2 \mathrm{~V}$ throughout these experiments, and ion image currents were collected and digitized in medium-resolution mode using 2.67-MHz bandwidth detection and $16 \mathrm{~K}, 32 \mathrm{~K}$, or $64 \mathrm{~K}$ data points.

The EID experiments were performed with the limonene molecular ion and the group of ions $\mathrm{W}(\mathrm{CO})_{n}^{+}$ ( $n=1-6)$ generated directly by electron impact. In each case, argon was maintained in the cell at a static pressure of $2 \times 10^{-6}$ torr, as measured with an uncalibrated Bayard-Alpert ionization gauge in an effort to cool any excited ions formed during the electron-impact ionization process. The typical background pressure of the system was $1 \times 10^{-9}$ torr. In the limonene studies, a quench pulse designed to remove any ions present in the cell initiated the experiment. Limonene vapor was then pulsed into the vacuum chamber by using a General Valve Series 9 pulsed solenoid valve [19]. Immediately after sample introduction, the electron beam was gated on for $5 \mathrm{~ms}$ to generate the molecular ion and fragment ions of limonene through electronimpact ionization. A nominal electron energy of $70 \mathrm{eV}$ produced the mass spectrum displayed in Figure 1; however, $16 \mathrm{eV}$ electrons were used for ionization in the EID experiments because the relative abundance of intact molecular ions is considerably higher (approximately by a factor of 2) under these conditions. After ionization, the electron gun was gated off, and swept double-resonance pulses [42] were used to isolate the limonene molecular ion, which was subsequently stored for $1 \mathrm{~s}$ in the cell in the presence of argon bath gas. This storage period served to cool any ions excited during the ionization and isolation processes and also ensured that any residual neutral limonene molecules introduced during the gas pulse were completely pumped away before EID. Experience with a variety of compounds indicates that the gas pulse is largely removed from the vacuum chamber

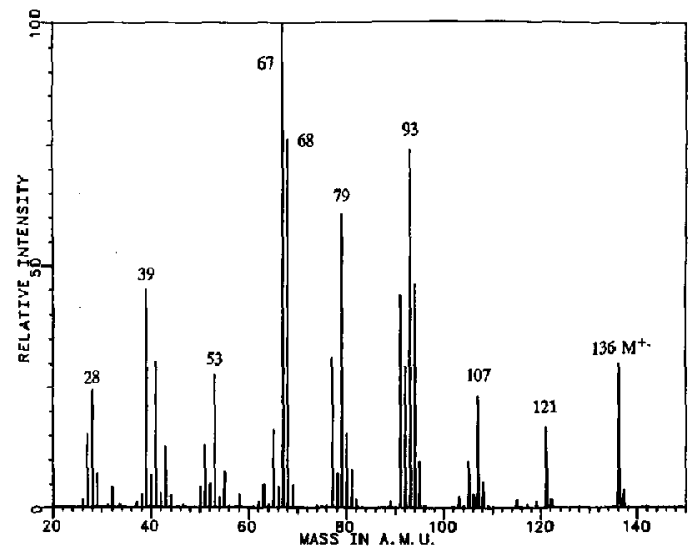

Figure 1. The $70-\mathrm{eV}$ electron-impact ionization mass spectrum of limonene. 
within approximately $0.7 \mathrm{~s}$. After this cooling interval, the electron gun was gated on again for $0.5 \mathrm{~s}$ at a nominal energy ranging from 3 to $5 \mathrm{eV}$, with an electron emission current of approximately $1 \mu \mathrm{A}$, inducing fragmentation of the limonene molecular ion. Finally, an EID mass spectrum was collected through broadband ion excitation and digitization of the resulting ion image currents.

To ensure that the fragmentation observed resulted exclusively from EID of the limonene molecular ion, two additional studies were performed. The first was designed to determine whether any residual limonene molecules from the gas pulse remained in the cell during the EID period and, if so, whether electronimpact ionization of these molecules was contributing to the observed fragmentation. To investigate this possibility, the entire sequence was performed with the electron gun gated off during the electron-impact ionization period so that no ions were formed. No fragment ions were observed when the electron gun was gated on later in the sequence during the EID period, demonstrating that no background gas was being ionized and fragmented during the EID period. Second, an experiment was designed to determine whether fragmentation of the limonene molecular ion during the 1-s cooling period was responsible for the EID mass spectrum. The sequence was performed again, this time with the electron gun gated on during the electron-impact ionization period but gated off during the EID period. Under these conditions, some fragmentation of the limonene molecular ion, arising presumably through CID with the background argon, was observed; however, this experiment clearly demonstrated that most of the fragmentation observed in the EID mass spectrum was the direct result of electron-ion collisions. The final EID mass spectrum was generated through background subtraction of the CID contribution (electron gun gated on during the ionization period and off during the EID period) from the mass spectrum observed with the electron gun gated on during both the ionization and EID periods. An EID mass spectrum generated in this manner with electrons of nominal energy $4 \mathrm{eV}$ is displayed in Figure 2.

A very similar pulse sequence was used to study EID of $\mathrm{W}(\mathrm{CO})_{n}^{+}(n=1-6)$. Once again a quench pulse initiated the sequence, and $W(\mathrm{CO})_{6}$ was introduced into the vacuum chamber by using a pulsed valve. A $10-\mathrm{eV}$ electron beam was gated on for $0.005 \mathrm{~s}$ during the electron-impact ionization period and then gated off. Unlike the limonene experiment, no swept doubleresonance isolation was performed in the $W(\mathrm{CO})_{6}$ experiment. The ions were then cooled for $1 \mathrm{~s}$, which also permitted residual neutrals from the gas pulse to be removed from the mass spectrometer. After this cooling interval, a variable period followed, during which time the electron beam either remained off (for the blank) or was gated back on (for EID). Figure 3a shows the mass spectrum obtained using a 0.01-s period with the electron gun gated off. The characteristic

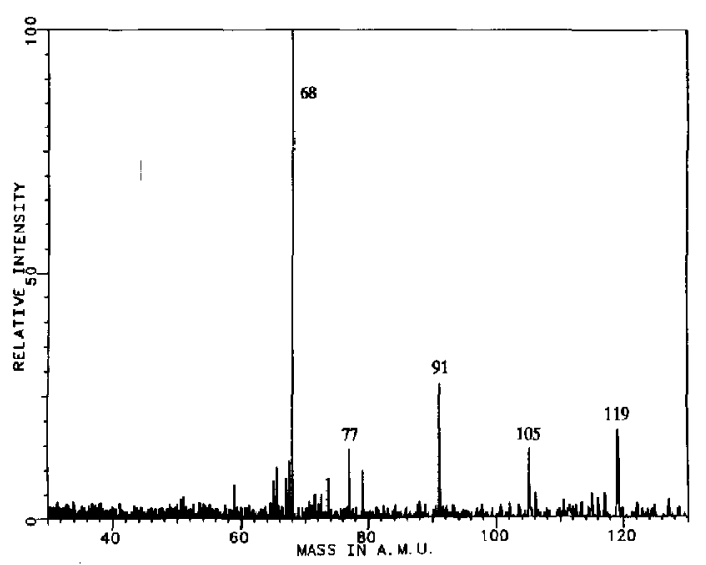

Figure 2. Background-corrected EID mass spectrum for the limonene molecular ion irradiated with $4-\mathrm{eV}$ electrons at an emission current of approximately $1 \mu \mathrm{A}$ for $0.5 \mathrm{~s}$.

isotope pattern is evident. The sequence was performed again, this time with the electron gun gated on for $0.01 \mathrm{~s}$ at $5 \mathrm{eV}$ and a $0.5-\mu \mathrm{A}$ emission current, producing the EID mass spectrum displayed in Figure $3 b$. Spectral subtraction of the data in Figure 3a from Figure $3 b$ yielded the background-corrected EID mass spectrum in Figure 3c.

The nominal electron energies quoted in this article represent the uncorrected values obtained by selecting a particular energy through the FTMS-2000 software. In practice, the actual electron energy is slightly reduced by the space charge potential of the electron beam. The reduction in electron energy $\Delta V$ is given by

$$
\Delta V=\frac{i_{c}}{5.93 \times 10^{7}\left(V_{0}\right)^{1 / 2} C}
$$

where $i_{e}$ is the electron beam current (in amperes), $5.93 \times 10^{7}$ the velocity of a $1.0-\mathrm{eV}$ electron (in centimeters per second), $V_{0}$ the nominal electron energy (in electron volts), and $C$ the capacitance per unit length between the electron beam and the cell (in farads) [43]. Although $\Delta V$ can be substantial under the conditions used in this study (on the order of $0.5 \mathrm{eV}$ ) [33], this represents only a small correction to the energy deposited during EID, because multiple electron-ion collisions are involved, and the energy of the exciting electron after the collision is undetermined. Therefore, this small correction to the electron energy was not performed in association with these experiments.

\section{Results}

\section{Limonene}

Our previous studies of limonene, as well as the $70-\mathrm{eV}$ electron-impact ionization mass spectrum displayed in Figure 1, clearly indicate that one of the predominant 

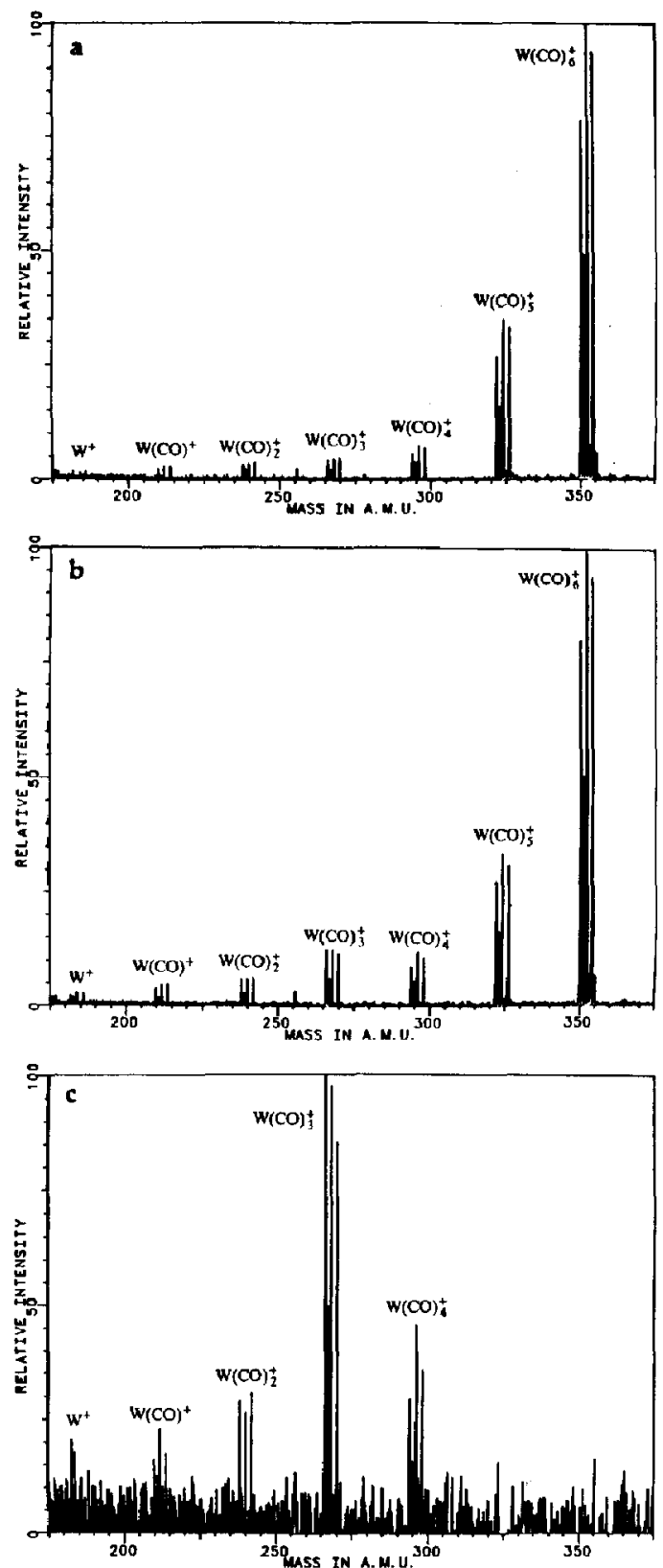

Figure 3. (a) The 10-eV electron-impact ionization mass spectrum of $\mathrm{W}(\mathrm{CO})_{6}^{+}$obtained by using a 1-s cooling time, followed by an additional 0.01-s period with the electron beam gated off. This spectrum serves as the blank. (b) Mass spectrum generated under otherwise identical conditions as (a), but with the electron gun gated on after the 1-s cooling time. The ions were irradiated with 5-eV electrons at an emission current of $0.5 \mu \mathrm{A}$ for $0.01 \mathrm{~s}$. (c) Background correction of the spectrum shown in (b) with the data in (a) produces the resulting EID mass spectrum.

fragment ions produced from limonene under electron-impact ionization has $m / z 68$ and arises from the limonene molectlar ion via a retro-Diels-Alder dissociation process [27, 44]. Surprisingly, charge- exchange ionization of limonene with a number of reagent ions, including the molecular cations of benzene, $\mathrm{CS}_{2}$, and $\mathrm{C}_{3} \mathrm{~F}_{6}$, as well as ionized argon and neon, produces little or no retro-Diels-Alder fragmentation. Furthermore, low-energy CID and photodissociation of the mass-selected limonene molecular ion also yield essentially no intensity at $m / z$ 68 [27]. In stark contrast, charge-exchange ionization with $\mathrm{Xe}^{+}$produces an abundant retro-Diels-Alder fragment ion. This observation, in conjunction with the electronimpact ionization results, suggests that the shape of the distribution of internal energies deposited during ionization and activation strongly affect the fragmentation of the limonene molecular ion $[27,44]$. With this information in mind, it is particularly interesting to examine the fragmentation data generated through EID of the limonene molecular ion. Electron-induced dissociation was performed at nominal electron energies of 3,4 , and $5 \mathrm{eV}$, and a background-corrected EID mass spectrum produced with 4 -eV electrons is displayed in Figure 2. The principal fragment ion observed is the retro-Diels-Alder species at $m / z$ 68. Electron-induced dissociation with $3-\mathrm{eV}$ electrons produces data with poor signal-to-noise characteristics, but only the retroDiels-Alder fragment ion is discernible, whereas this ion is the base peak in the 5-eV EID mass spectrum as well. The presence of the intense signal at the highly diagnostic $m / z 68$ in both the electron-impact mass spectrum and the EID mass spectra suggests that the distribution of internal energies deposited during EID overlaps in some specific fashion that associated with electron-impact ionization, although the distribution for the 70-eV electron-impact process is clearly broader and extends to higher energies.

\section{$\mathrm{W}(\mathrm{CO})_{6}$}

To further investigate the internal energy deposited during EID, we used a well-established technique based on the fragmentation of the "thermometer molecule," $W(\mathrm{CO})_{6}$. Electron-impact ionization of this molecule with $10-\mathrm{eV}$ electrons produced the mass spectrum displayed in Figure 3a. In an effort to enhance parent-to-product ion conversion efficiency, no swept double-resonance isolation was performed (see above). All of the ions in Figure $3 a$ were subjected to electron irradiation at a nominal energy of $5 \mathrm{eV}$ and an emission current of $0.5 \mu \mathrm{A}$, generating the mass spectrum in Figure 3b. Background subtraction provided the EID mass spectrum in Figure $3 c$.

By using a previously described algorithm [35] and the well-known appearance energies for the production of the various ions, $\mathrm{W}(\mathrm{CO})_{n}^{+}(n=0-6)$, the intensities of these ions can be converted to an internal energy distribution $P(\epsilon)$ versus $\epsilon$, as displayed in Figure 4 . Note that in this case the conversion includes an additional approximation because the algorithm assumes that $\mathrm{W}(\mathrm{CO})_{6}^{+}$is the exclusive precursor of the fragments. Although this is not the case, it is the dominant species. (Note, too, that appearance energies 


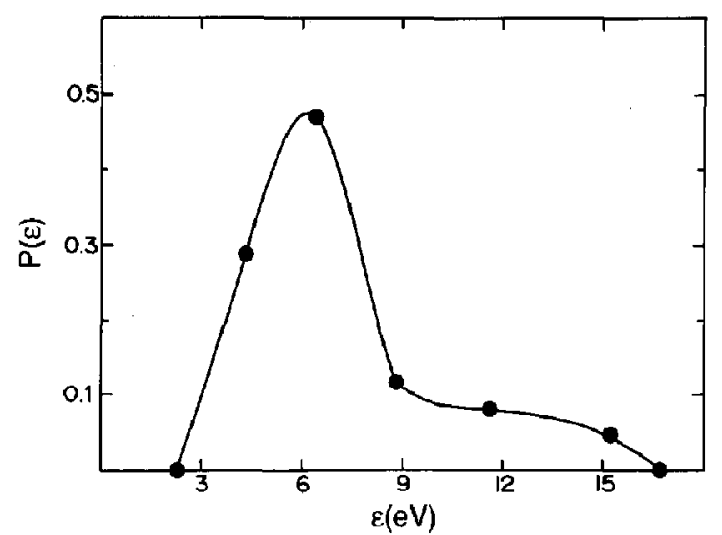

Figure 4. Internal energy distribution $P(\epsilon)$ versus $\epsilon$ for electron-ion collisions among 5-eV electrons at an emission current of $0.5 \mu \mathrm{A}$ and $\mathrm{W}(\mathrm{CO})_{n}^{+}(n=1-6)$ for $0.01 \mathrm{~s}$.

rather than ion enthalpies are used because these data allow correction for internal excitation carried by the neutral fragments [35].) Examination of Figure 4 clearly indicates that multiple electron-ion collisions are involved in EID under these conditions, so that the data in Figure 4 can be considered an effective internal energy distribution for the multistep activation process. The average internal energy deposited is $6.9 \mathrm{eV}$, which exceeds the nominal electron energy and therefore supports the notion of multiple electron-ion collisions. Of note, the distribution from EID shown in Figure 4 is similar to the $70-\mathrm{eV}$ electron-impact ionization internal energy distribution for $W(\mathrm{CO})_{6}$ displayed in Figure 5.

To further evaluate experimental control of energy deposition during EID, we investigated the effects of irradiation time on the fragmentation of $\mathrm{W}(\mathrm{CO})_{n}^{+}(n=$ 1-6). The nominal electron energy and emission current were maintained at $5 \mathrm{eV}$ and $0.5 \mu \mathrm{A}$, respectively, whereas the irradiation time was increased from $0.01 \mathrm{~s}$,

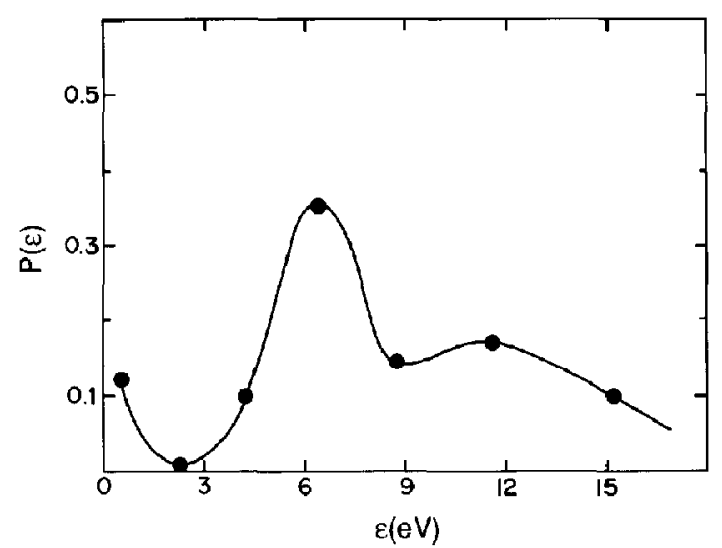

Figure 5. Internal energy distribution $P(\epsilon)$ versus $\epsilon$ for $70-\mathrm{eV}$ electron-impact ionization of $\mathrm{W}(\mathrm{CO})_{6}^{+}$. (From ref 35; adapted with permission from Elsevier Science Publishers.)
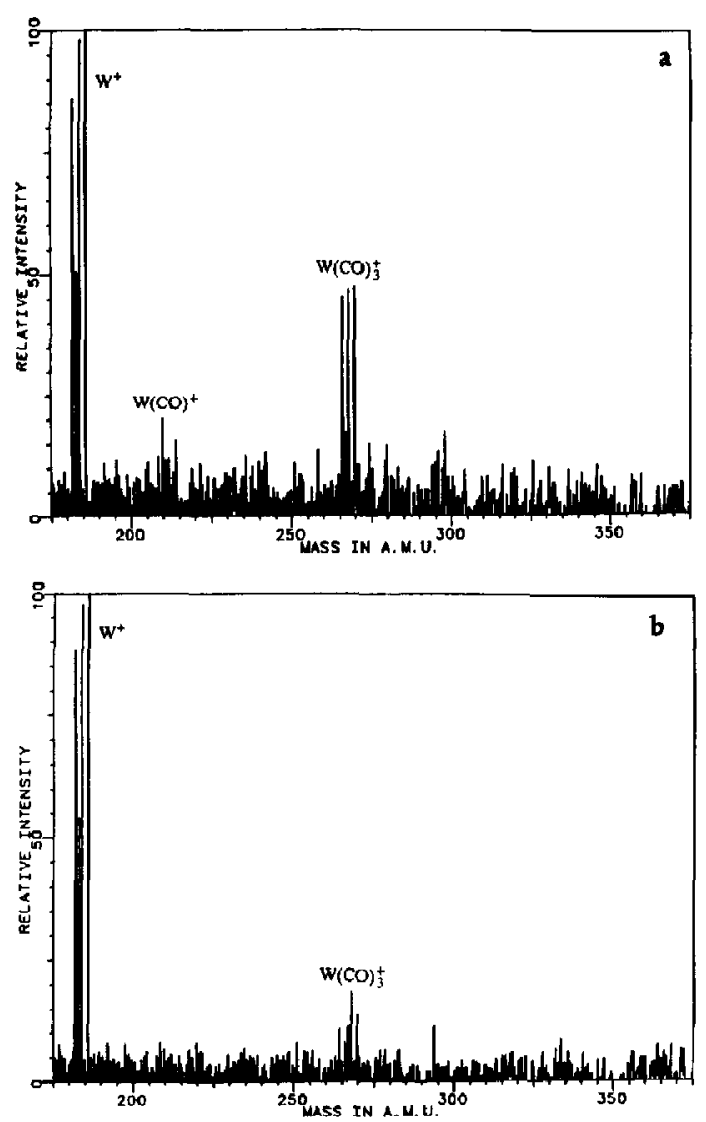

Figure 6. Background-corrected EID mass spectrum for $\mathrm{W}(\mathrm{CO})_{n}^{+}$ $(n=1-6)$ irradiated with 5-eV electrons at an emission current of $0.5 \mu \mathrm{A}$ for (a) $0.02 \mathrm{~s}$ and (b) $0.1 \mathrm{~s}$. Note that the blank spectrum used for background subtraction in each case was obtained under otherwise identical conditions, except that the electron beam remained gated off after the ionization pulse.

used to produce the EID mass spectrum in Figure 3c, to $0.02 \mathrm{~s}$ in Figure 6a and $0.1 \mathrm{~s}$ in Figure 6b. These data demonstrate that as the irradiation time is increased, additional electron-ion collisions occur, and the extent of fragmentation is enhanced. At $0.1 \mathrm{~s}$, essentially all of the ions undergoing repeated electron collisions are converted to $\mathrm{W}^{+}$through EID, although this represents a small fraction of the original $W(\mathrm{CO})_{6}^{+}$present. This process requires [35] $13.0 \mathrm{eV}(n=6), 11.8 \mathrm{eV}$ $(n=5), 9.6 \mathrm{eV}(n=4), 7.8 \mathrm{eV}(n=3), 5.5 \mathrm{eV}(n=2)$, and $2.9 \mathrm{eV}(n=1)$, indicative of the large internal energy depositions accessible through EID.

\section{Discussion}

The results of this study indicate that the internal energies deposited during EID under selected conditions are often similar to that of electron-impact ionization. Recent EID studies with $\mathrm{Fe}(\mathrm{CO})_{5}^{+}$and the $n$-butylbenzene molecular ion further support this contention [45]. This aspect of EID is consistent with the 
expectation that ion activation during electron-ion collisions must proceed through excitation of electronic modes of the ions. This makes EID an attractive complementary activation method to CID, which is believed to proceed via excitation of vibrational modes under the low-energy CID conditions used in FTICRMS [46, 47], although notable exceptions to this contention have been reported $[48,49]$. The amount of energy deposited can be selected with both EID and CID, so that studies comparing these two techniques could be aimed at investigating underlying differences in ion fragmentation arising from the dynamics of energy deposition rather than the amount of energy deposited.

This study also points out that control of ion excitation with EID can be achieved in a number of ways. Increased electron energy enhances fragmentation, as described in early studies on benzonitrile [33]. The same trend is observed as the electron energy is increased from 3 to $5 \mathrm{eV}$ during EID of the limonene molecular ion. Similar increases in fragmentation are induced when the number of electron-ion collisions is increased, either through longer irradiation times or higher electron emission currents $[20,50,51]$, as observed in this study with $\mathrm{W}(\mathrm{CO})_{n}^{+}(n=1-6)$ and in a similar study by Kerley et al. [34] using $\mathrm{Fe}(\mathrm{CO})_{5}^{+}$.

A final word must be added to this report to address the conversion efficiency of the EID process. Only a small amount of dissociation [ $\leq 5 \%$; obtained by taking the sum of the absolute intensities of the product ions in Figure $3 \mathrm{c}$ and dividing it by the absolute intensity of $\mathrm{W}(\mathrm{CO})_{6}^{+}$in Figure $3 a$ ] took place during the 0.01-s irradiation time used in that experiment. Depending on the conditions, between $5 \%$ and $85 \%$ conversion has been reported in earlier studies [20, 28, 31]. The primary limitation on conversion efficiency with EID is the fact that the ions of interest diffuse away from the center of the cell in a relatively short time owing to space charge effects as well as the shape of the electrostatic trapping field used [52, 53]. Because the electron beam in the FTMS-2000 is directed through the center of the cell, this ion diffusion results in reduced overlap between the electron beam and the ion cloud and limits EID conversion efficiency. In this sense, the most significant limitation of the experiment is the fact that a delay period of the order of $1 \mathrm{~s}$ is required to ensure that all of the gas introduced via the pulsed valve is pumped away before initiating EID. This experiment might be improved by using an FIICRMS configured with a dual cell [28], so that ions could be formed under relatively highpressure conditions in the source cell, cooled for $1 \mathrm{~s}$, and then transferred to the low-pressure analyzer cell for immediate EID. Conversion efficiencies of the transferred ions under these conditions are expected to be significantly improved, because these ions necessarily must be in the center of the cell to pass through the 2-mm conductance limit, and the 1-s cooling time occurs before transfer. Another important concern for maintaining high conversion efficiency involves swept double-resonance isolation. Off-resonance excitation of the ions of interest during their isolation can increase the radii of their cyclotron orbits enough to remove them from the path of the electron beam. This problem becomes more significant as the mass of the ions to be ejected approaches that of the ion to be isolated, particularily at higher mass where resolution is decreased. For example, the limonene cation was isolated and required a 0.5-s EID time to achieve about the same amount of dissociation as that seen for $W(\mathrm{CO})_{6}^{+}$, which was not isolated and required only a 0.01-s EID time. Although the cross sections are certainly different, the longer EID time needed for limonene is due in part to off-resonance excitation during the isolation step. An alternative solution could be realized by using a tailored excitation method, such as stored waveform inverse Fourier transform (SWIFT) excitation [54], to achieve ion isolation.

\section{Conclusions}

This study demonstrates once again that EID is a viable alternative to other ion activation techniques. This technique promises to produce diagnostic fragmentation, perhaps even for large biomolecular ions [20]. Furthermore, control of the extent of excitation is achieved in a straightforward manner through selection of the electron energy, the electron flux or emission current, and the irradiation time during which electron-ion collisions occur.

As practiced in FTICRMS, EID enjoys a number of additional advantages as well. First, the problematic increased gas load required for CID is not encountered in EID. The relatively high pressure of the argon bath gas, used in this study to cool any excited ions formed during the electron-impact ionization process, is not necessary for routine EID experiments or highresolution studies [20, 28]. Second, only ions that interact with the electron beam produce fragment ions, and the electron beam is directed through the center of the cells in a dual-cell instrument, so that the fragment ions generated during EID are optimally positioned for subsequent transfer between the cells. Finally, EID promises to provide information complementary to low-energy CID, because the dynamics of activation are different in the two techniques, EID proceeding via electronic excitation and $\mathrm{CID}$ proceeding via vibrational excitation.

\section{Acknowledgments}

BSF acknowledges the continuing support of the National Science Foundation (CHE-8920085) for development of FTICRMS methodology. RGC acknowledges the support of the National Science Foundation (CHE-8721768). JRG acknowledges the Analytical Division of the American Chemical Society and the Eastman Kodak Company for fellowship support. In addition, the authors thank Extrel for the single cell used in our FTMS-2000 during the course of these experiments. 


\section{References}

1. McLuckey, S. A.; Sallans, L.; Cody, R. B.; Burnier, R. C.; Verma, S.; Freiser, B. S.; Cooks, R. G. Int. J. Mass Spectrom. Ion Phys. 1982, 44, 215.

2. Williams, D. H.; Howe, I. Principles af Organic Mass Spectrometry; McGraw-Hill: London, 1972; Chapter 4.

3. Hillenkamp, F. Presented at the 38th ASMS Conference on Mass Spectrometry and Allied Topics; Tucson, AZ, June 1990; pp. 158 and 160.

4. Fenn, J. B.; Mann, M.; Meng, C. K.; Wong, S. F.; Whitehouse, C. M. Science $1989,246,64$.

5. Ruepstorff, P. Acc. Chem. Res. 1989, 22, 421.

6. McFarlane, R. D. Anal. Chem. 1983, 55, 1247A

7. Alexander, A. J.; Thibault, D.; Boyd, R. K. J. Am. Chem. Soc. 1990, $112,2484$.

8. Biemann, K. Anal. Chem. 1986, 58, 1288A.

9. Cooks, R. G. Ed. Colision Spectroscopy; Plenum: New York, 1978.

10. Mabud, M. A.; DeKrey, M. J.; Cooks, R. G. Int. J. Mass Spectrom. Ion Proc. 1985, 67, 285.

11. Bowers, M. T., Ed. Gas Phase Ion Chemistry; Vol. 3; Academic Press: New York, 1984.

12. Cody, R. B.; Freiser, B. S. Int. J. Mass Spectrom. Ion Phys. 1982, 41, 199.

13. Williams, E. R.; Henry, K. D.; McLafferty, F. W.; Shabanowitz, J.; Hunt, D. F. J. Am. Soc. Mass Spectrom. 1990, 1, 413.

14. Dunbar, R. C. In Gas Phase Ion Chemistry; Vol. 3; Bowers, M. T., Ed.; Academic Press: New York, 1984; Chapter 20.

15. Urlando, K.; Fenselau, C.; Cotter, R. J. J. Am. Soc. Mass Spectrom. 1991, 2, 189.

16. Gord, J. R.; Freiser, B. S. Anal. Chim. Acta 1989, 225, 11.

17. Chaderi, S.; Littlejohn, D. P. Presented at the 33rd ASMS Conference on Mass Spectrometry and Allied Topics; San Diego, CA, 1985; p. 727.

18. Wise, M. B. Anal. Chem. 1987, 59, 2289.

19. Carlin, T. J.; Freiser, B. S. Anal. Chem. 1983, 55, 571.

20. Wang, B.-H.; McLafferty, F. W. Org. Mass Spectrom. 1990, 25, 554.

21. Freiser, B. S. Int. J. Mass Spectrom. Ion Phys. 1980, 33, 263.

22. Dolder, K. T.; Harrison, M. F. A.; Thonemann, P. C. Proc. R. Soc. London 1963, A274, 546.

23. Dolder, K. T.; Harrison, M. F. A.; Thonemann, P. C. Proc. R. Soc. London 1961, A264, 367.

24. Van Zyl, B; Dunn, G. H. Phys. Rev. 1967, 163, 43.

25. Dunn, G. H.; Van Zyl, B. Phys. Rev. 1967, 154, 40.

26. Dunn, G. H.; Van Zyl, B.; Zare, R. N. Phys. Rev. Lett. 1965, 15, 610.

27. Horning, S. R.; Wood, J. M.; Gord, J. R.; Freiser, B. S.; Cooks, R. G. Int. J. Mass Spectrom. Ion Proc. 1990, 101, 219.
28. Cody, R. B.; Freiser, B. S. Anal. Chem. 1987, 59, 1056.

29. Tajima, S.; Tobita, S.; Ogino, K.; Niwa, Y. Org. Mass Spectrom. 1986, 21, 236.

30. Fedor, D. M.; Cody, R. B.; Burinsky, D. J.; Freiser, B. S.; Cooks, R. G. Int. J. Mass Spectrom. Ion Phys. 1981, 39, 55.

31. Cody, R. B.; Freiser, B. S. Anal. Chem. 1979, 51, 547.

32. Freiser, B. S. Int. J. Mass Spectrom. Ion Phys. 1978, 26, 39.

33. Freiser, B. S.; Beauchamp, J. L. Chem. Phys. Lett. 1976, 42, 380.

34. Kerley, E. L.; Hanson, C. D; Russcl1, D. H. Anal. Chem. 1990, $62,409$.

35. Wysocki, V. H.; Kenttämaa, H. I.; Cooks, R. G. Int. I. Mass Spentrom. Ion Proc. 1987, 75, 181.

36. Wysocki, V. H.; Kenttämaa, H. I.; Cooks, R. G. J. Phys. Chem. $1988,92,6465$.

37. Horning, S. R.; Vincenti, M.; Cooks, R. G. J. Am. Chem. Soc. $1990,112,119$.

38. Horning, S. R.; Cooks, R. G.; Vincenti, M. Adv. Mass Spectrom. 1989, 11, 788.

39. DeKrey, M. J.; Kenttämaa, H. I.; Wysocki, V. H.; Cooks, R. G. Org. Mass Spectrom. 1986, 21, 193.

40. Cooks, R. G.; Ast, T.; Kralj, B.; Kramer, V.; Žigon, D. J. Am. Soc. Mass Spectrom. 1990, 1, 16.

41. Freiser, B. S. Chemtracts 1989, 1, 65.

42. Comisarow, M. B.; Grassi, V.; Parisod, G. Chem. Phys. Lett. $1978,57,413$.

43. Haeff, A. V. Proc. IRE 1939, 27, 586.

44. Vincenti, M.; Horning. S. R.; Cooks, R. G. Org. Mass Spectrom. 1988, 23, 585.

45. Cody, R. B. Personal communication, 1990.

46. Singh, S.; Harris, F. M.; Boyd, R. K.; Beynon, J. H. Int. J. Mass Spcctrom. Ion Proc. 1985, 66, 131.

47. Durup, J. In Recent Developments in Mass Spectroscopy; Ogata, R.; Hayakaya, H., Eds.; University Park Press: Tokyo, 1970; p. 921.

48. Qian, K.; Shukla, A.; Howard, S.; Anderson, S.; Futrell, J. J. Phys. Chem. 1989, 93, 3889.

49. Shukla, A. K.; Qian, K.; Howard, S. L.; Anderson, S. G.; Sohlberg, K. W.; Futrell, J. H. Int. J. Mass Spectrom. Ion Prac. $1989,92,147$.

50. Freiser, B. S.; Beauchamp, J. L. Chem. Phys. Lett. 1976, 42, 380.

51. Cody, R. B.; Freiser, B. S. Anal. Chem. 1982, 51, 547.

52. Francl, T. J.; Fukuda, E. K.; Mclver, R. T. Jr. Int. J. Mass Spectront. Ion Phys. 1983, 50, 151.

53. Sharp, T. E.; Eyler, J. R.; Li, E. Int. 1. Mass Spectrom. Ion Phys. 1972, 9, 421.

54. Marshall, A. G.; Wang, T.-C.; Ricca, T. L. J. Am. Chem. Soc. 1985, 107, 7893 . 